\title{
Transplant Glomerulopathy: Morphology, Associations and Mechanism
}

\author{
James Fotheringham ${ }^{\mathrm{a}}$ Carole A. Angel ${ }^{\mathrm{b}}$ William McKane ${ }^{\mathrm{a}}$ \\ aSheffield Kidney Institute and bepartment of Histopathology, Sheffield Teaching Hospitals NHS Foundation Trust, \\ Sheffield, UK
}

\section{Key Words}

Kidney transplantation - Glomerulopathy $\cdot \mathrm{C} 4 \mathrm{~d} \cdot$ Chronic antibody-mediated rejection

\begin{abstract}
Transplant glomerulopathy (TG) is a lesion with specific morphology and strong evidence of an immune mechanism. The incidence of TG is approximately $20 \%$ by 5 years after transplantation. TG is characterized by proteinuria, hypertension and declining graft function. Appearances on light microscopy include thickened capillary walls and double contours, with reduplication or lamination of the glomerular basement membrane on electron microscopy. TG is associated with acute rejection, the antibody status before transplantation and de novo HLA antibodies. HLA class II and/or donor-specific antibodies incur additional risks. Desensitization protocols do not always prevent the development of TG in highly sensitized individuals. Associations between TG, past or current $\mathrm{C} 4 \mathrm{~d}$ and the presence of alloantibodies are recognised, however, C4d in the peri-tubular capillaries or glomeruli is not a prerequisite at the time of diagnosis. Clinical observation and animal models suggest that TG arises as a consequence of chronic endothelial cell (EC) injury by the humoral arm of the immune system. In some cases, this follows a period of EC accommodation after an episode of acute injury. Proposed treatments include augmentation of background immunosuppression, and trials of monoclonal therapies targeted at CD20-positive B cells are underway.
\end{abstract}

Copyright $\odot 2009$ S. Karger AG, Basel

\section{Introduction}

In 2005, the Banff group eliminated the term chronic allograft nephropathy [1], based on concerns that the transplant community had adopted it as a specific disease entity and abused it as a mechanistic label for interstitial fibrosis and tubular atrophy (IF/TA). Transplant glomerulopathy (TG) has survived as a lesion with specific morphology and strong association with humoral immunity. This paper reviews the morphology of TG, its clinical impact and its probable immune mechanisms.

\section{Morphology}

The classic lesion of TG is distinctive and easily recognisable. On light microscopy, there are thickened capillary walls and double contours demonstrable on Periodic-acid Schiff and/or silver stains (fig. 1a). The extent of double contouring forms the basis for grading in the Banff classification [2]. There may or may not be increased mesangial matrix and glomerular cellularity. Using electron microscopy, there is reduplication or lamination of the glomerular basement membrane (GBM) and some cases show expansion of the lamina rara interna with deposition of finely fibrillary or flocculent material in a subendothelial location. Extensions of mesangial and/or endothelial cell (EC) cytoplasm are usually seen in the expanded subendothelial zone (fig. 1b). Early stages of TG can sometimes be recognised and were described

\section{KARGER \\ Fax +41613061234 E-Mail karger@karger.ch} www.karger.com
Dr. James Fotheringham

Sheffield Kidney Institute, Northern General Hospital

Herries Road

Sheffield S5 7AU (UK)

Tel. +44 114271 4808, Fax +44 114271 4604, E-Mail james.fotheringham@nhs.net 


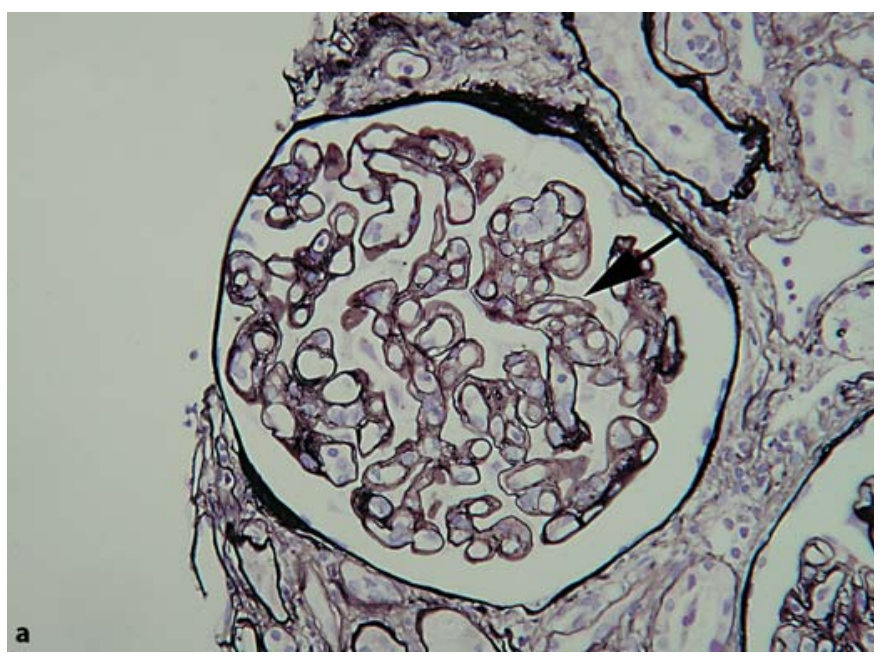

Fig. 1. Histopathology of TG. a Methenamine silver showing double contouring of glomerular capillary walls (arrow, $\times 400$ ). b Electron micrograph showing lamination of GBM, reactive EC with cytoplasmic extension into the neo-basement membrane material and a mononuclear cell in the lumen $(\times 8,000)$. c C4d expression in glomerular capillaries in TG in the absence of PTC staining $(\times 250)$.

over 20 years ago [3]. On light microscopy, EC and mesangial cells appear swollen and the capillary lumen is reduced. Silver stains show vacuolation or reticulation, corresponding to the early lamination/reduplication seen at electron microscopy.

TG must be distinguished from other glomerulopathies, in particular ischaemia, thrombotic micro-angiopathy (TMA) and recurrent or de novo immune complex glomerulopathy. Ischaemic glomeruli have wrinkled capillary walls rather than double contours but it can be impossible to distinguish between TG and TMA on morphological grounds alone and clinical correlation is necessary. TMA, a lesion seen in native renal biopsies in conditions such as haemolytic uraemic syndrome and lupus nephritis can also occur in the renal transplant and is a recognised rare complication of calcineurin inhibitors. Microthrombi may sometimes be observed in acute
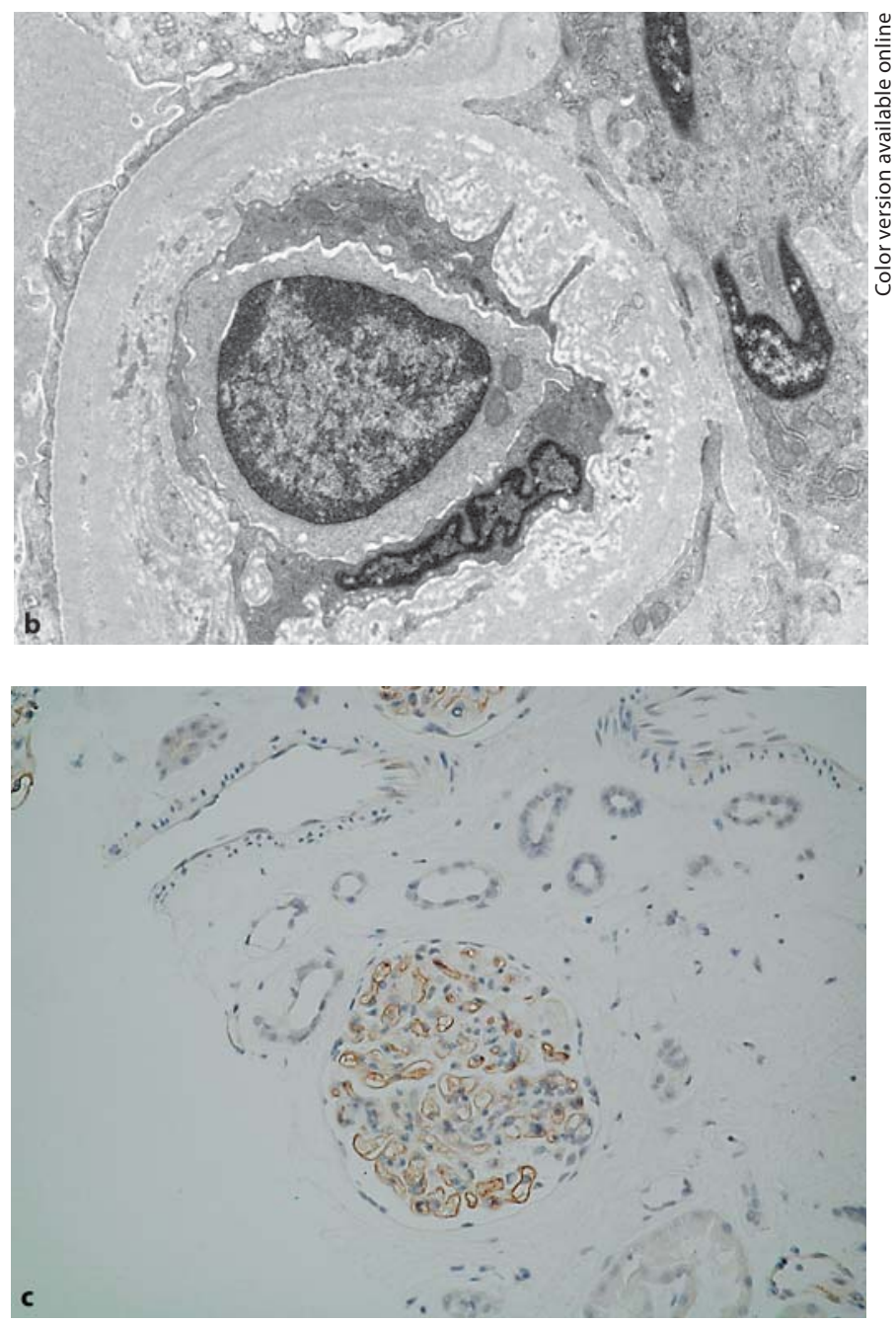

antibody-mediated rejection [4], suggesting that repeated antibody-mediated episodes of TMA resulting in endothelial damage may play a role in the development of TG.

Immune complex glomerulonephritis can be diagnosed using immunohistology and/or electron microscopy. Cases of otherwise typical TG, in which immune deposits are found, are usually excluded from studies of TG but it is possible that such cases represent an alternative phenotype in which anti-HLA antibodies themselves may be deposited [5]. We recently reported a temporal association between anti-HLA antibody, de novo immune complex glomerulopathy and histological evidence of antibody-mediated damage. We hypothesized that the glomerulopathy in this case might be an atypical presentation of chronic antibody-mediated rejection [6]. 


\section{Clinical Characteristics}

The incidence of TG was reported in a retrospective study of protocol and clinically indicated biopsies [7]. Rare before 6 months, the incidence increases from $4.0 \%$ at 1 year to $20.2 \%$ at 5 years. Unsurprisingly, with the exclusion of clinically indicated biopsies, the incidence in this report dropped to $2.8 \%$ at 1 year and $11.5 \%$ at 5 years. Importantly, the group identified that the lesion is progressive, with the TG grade (cg0-3) worsening in a third of the patients who were re-biopsied. One large study reviewing clinically indicated biopsies alone reported a $5.1 \%$ incidence [5]. The mean time from transplantation to diagnosis ranges from 2.75 to 8.2 years in clinically indicated biopsy studies $[7,8]$ and is likely to be influenced by graft surveillance and biopsy thresholds of the transplanting centre.

TG is characterized by proteinuria, declining graft function and hypertension. Declining function often lags behind the development of the histopathologic lesion. Indeed, in the study by Gloor et al. [7] 47\% of patients had a serum creatinine $<132 \mu \mathrm{mol} / \mathrm{l}$. Other studies have mainly reviewed clinically indicated biopsies, and reported poorer graft function at the time of biopsy [5]. Proteinuria is the hallmark of glomerular injury and a relationship with TG would be anticipated. TG-positive protocol biopsies at 1 year are associated with significantly more proteinuria than non-TG biopsies ([7] and proteinuria is frequently a feature of TG in reviews of clinically indicated biopsy series $[5,8]$ ).

\section{Clinical and Laboratory Associations}

\section{Rejection}

TG is more commonly seen in patients with historical or ongoing acute rejection. Sis et al. [5] reported a high incidence of previous rejection (54\%), often multiple (40\%), in their clinically indicated biopsy study; $71 \%$ of patients had experienced T-cell-mediated rejection and $26 \%$ antibody-mediated rejection. Also relevant is the presence of ongoing immune injury. Studies of clinically indicated biopsies have demonstrated raised scores for glomerulitis, tubulitis and peritubular capillary (PTC) inflammation according to the Banff 97 criteria [5]. In protocol studies, increasing cg scores were associated with increasing amounts of glomerulitis, however, tubulitis scores did not significantly alter [7].

\section{C4d}

The association between PTC binding of the complement product of the classical C4d pathway and acute antibody-mediated rejection is now well established [9]. Many studies have shown PTC staining in a proportion of cases with TG [8-10] and Regele et al. [10] were able to show that this association was highly statistically significant ( $p<0.0001)$. However, some cases of TG are PTC $\mathrm{C} 4 \mathrm{~d}$ negative. At present, the significance of $\mathrm{C} 4 \mathrm{~d}$-negative cases is uncertain; no statistically significant differences between $\mathrm{C} 4 \mathrm{~d}+$ and $\mathrm{C} 4 \mathrm{~d}-$ cases have yet been shown [5]. It should be noted that longitudinal biopsy results suggest marked variability in the presence of C4d over time [10], even in the ongoing presence of donor-specific antibody (DSA).

Glomerular C4d staining in TG cannot be properly assessed on frozen sections using the most widely used commercially available monoclonal anti-C4d antibody (Quidel ${ }^{\circledR}$ ) since positive staining of the mesangium and capillary walls is almost invariably present in normal glomeruli [11]. When a polyclonal antibody is used (Oxford Biomedica) on paraffin-embedded sections, however, this staining is abolished in normal glomeruli, although staining of PTCs is broadly comparable [11]. Thus, this technique can be used to investigate pathologically significant C4d staining in TG. It seems clear that the glomeruli in at least a proportion of TG cases show positive staining (fig. 1c), and in one study, segmental glomerular capillary staining was seen in 6 cases in the absence of PTC staining [10].

\section{HLA Antibodies}

There has been much recent interest in the presence of HLA antibodies and the development of TG. The presence of HLA antibodies prior to transplantation (presensitised patients) has been found to correlate with the development of TG [7] in a group of 582 transplant recipients with a negative T-cell complement-dependent cytotoxicity crossmatch before transplantation, even after multivariate analysis. These findings suggest that TG is a lesion that has a primary immunological aetiology, unlike IF/TA where no specific mechanism of injury is implied. It is now acknowledged that the development of IF/TA is a consequence of combined injuries, many of which are non-immune, such as calcineurin inhibitor toxicity.

Class II antibodies are found in conjunction with TG with greater frequency than their class I counterparts [5, 7]. Incremental levels of the risk of developing TG may exist, with class I being the lowest, ranging through class 
II non-DSA (NDSA) to class II DSA [7]. This study suggests that class I antibodies confer no greater risk than the absence of antibody, although this sub-analysis does not take donor specificity into account.

Desensitization protocols for recipients with high levels of HLA antibodies have become more widespread over the past 10 years. It is possible to reduce the incidence of acute antibody-mediated rejection in patients with DSA before transplantation using a variety of techniques including plasma exchange, intravenous immunoglobulin (IVIG) or anti-B-cell therapies such as rituximab. Although short-term outcomes are now excellent, there is growing concern that a high incidence of TG ensues. Recent data presented in abstract form report a TG incidence of $43 \%$ in patients treated with plasma exchange and IVIG, despite $83 \%$ of the patients achieving a negative crossmatch at transplantation [12]. The high incidence of TG in desensitization protocols argues against the likelihood of long-term stable accommodation (a stable graft in the presence of DSA).

The association between TG and de novo HLA antibodies also supports the concept of an aetiological link between TG and humoral immunity. The development of de novo DSA and NDSA is associated with more acute rejection, graft failure and proteinuria when compared to those who did not develop HLA antibodies [13]. DSA is also associated with more acute rejection and graft failure than NDSA. Among 25 patients in this study who had DSA and a complete annual screening record, 18 (72\%) developed TG and they had heavier proteinuria than those with NDSA. In our centre, where HLA antibody screening is performed at least annually, significantly more proteinuria developed in DSA compared with NDSA or a control group. Patients sensitized at the time of transplantation were excluded in this study and the proteinuria preceded the detection of DSA by 6 months [14]. This supports the hypothesis that early in the process of immune injury including TG development, HLA antibody is bound in the graft and undetectable in the serum. It also suggests that accommodation may not always be long lasting.

\section{Non-HLA Antibodies}

Following on from the discovery of antibodies to GBM antigens in the F344 to LEW rat renal transplant model for chronic rejection, Joosten et al. [15] analysed the sera of 16 patients collected at the time of biopsy subsequently showing TG. When compared to a control group, significantly more $(\mathrm{p}=0.0044)$ patients in the TG group had IgG anti-GBM antibodies. These anti-GBM did not bind to the well-characterized Goodpasture antigen $(\mathrm{NC1} \alpha 3$ chain of collagen type IV) but to the heparan sulphate proteoglycan agrin (44\%). The presence of anti-GBM antibodies was associated with more rejection episodes ( $\mathrm{p}=$ $0.0008)$, but no more proteinuria $(\mathrm{p}=0.2841)$. This was despite alterations in the structure of heparan sulphate proteoglycans leading to structural GBM changes. Other organs in which agrin is found were unaffected, and agrin has subsequently been identified as dispensable in maintaining GBM function, potentially explaining the relative absence of proteinuria in a previous study [16].

Setoguchi et al. [17] found a higher incidence of TG in the protocol biopsies of $48 \mathrm{ABO}$-incompatible recipients when compared to $133 \mathrm{ABO}$-compatible controls, although it did not reach statistical significance ( $15 \mathrm{vs.} 7 \%$, $p=0.57$ ), despite a higher incidence of C4d (75 vs. $32 \%$, $\mathrm{p}=0.02$ ). Detectable HLA antibodies and a history of antibody-mediated rejection were associated with TG.

\section{Chronic Antibody-Mediated Rejection}

The Banff group recognized the importance of the above associations in their 2005 report, classifying the diagnostic triad of late or chronic antibody-mediated rejection as (1) morphological features including TG and/or PTC basement membrane multi-layering (PTCBMML), (2) diffuse C4d deposition in the PTC and (3) the presence of DSA [1]. C4d deposition is cited as the best in situ evidence for an active humoral immunological process. Some flexibility exists, however, with TG/PTCBMML and either C4d or DSA allowing the diagnosis 'suggestive of chronic antibody-mediated rejection'.

The association between some of the above findings is best illustrated by Sis et al. [5], introducing an ABCD nomenclature combining the presence of HLA antibodies (A), PTCBMML (B) and PTC C4d deposition (C). TG was defined by double contours of GBM (D). The incidence of the complete $A B C D$ phenotype was $36 \%$ with a spectrum of the other possible incomplete phenotypes occurring less frequently; $69 \%$ of patients with appropriate sera and staining had concurrent HLA antibody (A) and/or C4d (C) in the presence of TG (D). The presence of C4d was strongly associated with the presence of HLA antibody $(\mathrm{p}=0.004)$, in a group with a high incidence of DSA $(85 \%$ of antibodies detected).

\section{Animal Studies}

Two recently published animal studies have improved our understanding of the aetiology and natural history of TG. Koch et al. [18] have adapted the F344 to LEW rat 


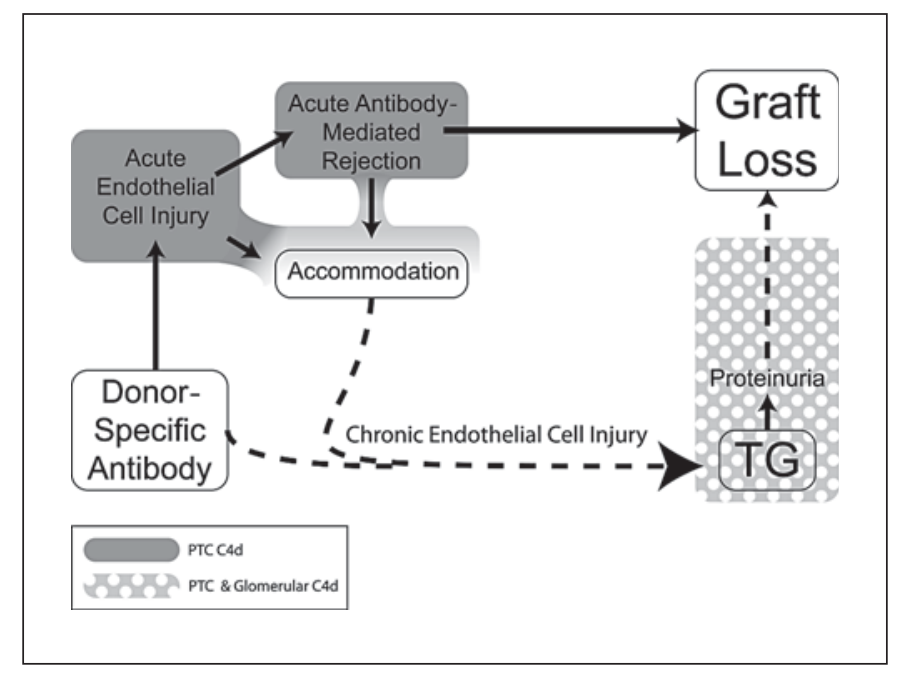

Fig. 2. Relationship between alloantibody, accommodation and TG. DSA activates EC in the allograft. Acute activation by hightitre antibody results in acute antibody-mediated rejection. EC accommodation affords protection against EC injury by DSA but may not last indefinitely. In at least some cases, chronic EC activation by DSA over many years leads to TG, proteinuria and graft failure. C4d staining of PTC is a reliable biomarker of acute humoral injury. C4d staining (glomeruli and PTC) supports a diagnosis of chronic humoral injury but its presence and intensity are variable.

renal transplant model to dissect out the role of humoral immunity in a passive transfer experiment. T-cell-deficient Lewis rats received allografts mismatched at the MHC Ib locus. Recipient rats infused with a monoclonal antibody against MHC Ib developed massive proteinuria and severe glomerulopathy, whereas controls did not. A cynomolgus monkey model describes the natural history of TG development based on the analysis of 417 specimens from 143 animals [19]. The monkeys received renal allografts using mixed chimaerism protocols but no maintenance immunosuppression. In results analogous to the clinical ABCD study described above [5], 48\% developed DSA, $29 \%$ C4d and $22 \%$ TG. The authors propose a sequential deterioration in graft histopathology and function initiated by DSA.

\section{Working Hypothesis}

We can see from the clinical and animal studies that a working hypothesis is developing that describes the mechanistic relationship between humoral immunity, accommodation and the development of TG (fig. 2). Activation of the humoral arm of the alloimmune response produces DSA. The target molecules are expressed on the EC surface which becomes activated and injury ensues. In some circumstances, perhaps related to the titre of the antibody [20], the outcome is acute humoral rejection and possible early graft loss. Accommodation is a term that describes an alternative outcome, whereby EC adapt to the humoral attack and graft function remains stable. The subsequent development of TG, in the context of DSA and morphologic features of chronic EC injury throughout the renal capillary bed, suggests that accommodation does not always result in long-term protection of the graft. Thus, GBM double contours and PTCBMML are considered the consequence of chronic EC injury by DSA. TG may or may not follow an acute episode, and although strongly associated with DSA, staining with C4d in PTC or glomeruli is not a prerequisite at the time of diagnosis. TG causes proteinuria and in common with all proteinuric renal diseases it progresses to graft failure. Most of the DSA described in detail are directed against mismatched HLA molecules but this need not always be the case and our understanding may be biased by the lack of widely available techniques to detect non-HLA antibody.

\section{Therapy}

Acute humoral rejection is now considered a treatable lesion. Therapies include antibody removal, IVIG and rituximab. Is it logical to go for antibody removal strategies in TG? The protracted time course of the injury argues against acute removal strategies. It is proposed, based on experience with case series, that a change in background immunosuppression can stabilize chronic humoral rejection [21]. Theruvath et al. [21] switched 4 patients with circulating DSA and TG with widespread PTC C4d deposits and declining graft function from a predominantly cyclosporine-based regime to tacrolimus and mycophenolate mofetil. They noted a rapid and sustained decrease in antibody titres and C4d deposition at 12 months. This strategy seems reasonable and is widely adopted but has not been subjected to a formal clinical trial. Furthermore, no benefit was observed when this approach was adopted for a subsequent series of 11 patients in another centre [22]. Despite the conflicting evidence and lack of a robust study, conversion to tacrolimus and mycophenolate is considered by many clinicians to be an appropriate therapy and forms the comparator in ongoing trials for chronic antibody-mediated rejection. Typical best supportive care for proteinuric chronic kidney dis- 
ease, including tight blood pressure control and blockade of the rennin-angiotensin system, also seems sensible. However, as this is an immunological disease a formal trial of an immunotherapy is required. Ongoing studies are currently assessing the role of rituximab, a chimaeric monoclonal antibody against CD20 that depletes B cells. It is hypothesized that rituximab may be influencing the antigen-presenting function of $\mathrm{B}$ cells, as it does not deplete the plasma cells that secrete alloantibody. Encouraging results have been observed in a small number of patients in published [23] and unpublished series. RituxiCAN-C4 (ClinicalTrials.gov Identifier NCT00476164) is an ongoing randomized controlled trial of standard therapy (tacrolimus and mycophenolate mofetil) versus rituximab and standard therapy in the UK and it is likely that other such studies are planned elsewhere.

\section{Conclusions}

TG is a relatively common finding in allograft biopsies and has a clear morphologic definition. Unlike other features seen in late allograft biopsies, it is a specific marker of immunologic attack. Animal and clinical studies support the hypothesis that chronic humoral injury is the cause of TG. Proteinuria and subsequent graft failure follows the development of TG and at present there is no clear evidence of the best therapeutic approach. It is hoped that ongoing trials of therapies targeted at the humoral immune response will improve outcomes for patients with TG in the future.

\section{References}

1 Solez K, Colvin RB, Racusen LC, Sis B, Halloran PF, Birk PE, Campbell PM, Cascalho M, Collins AB, Demetris AJ, Drachenberg CB, Gibson IW, Grimm PC, Haas M, Lerut E, Liapis H, Mannon RB, Marcus PB, Mengel M, Mihatsch MJ, Nankivell BJ, Nickeleit V, Papadimitriou JC, Platt JL, Randhawa P, Roberts I, Salinas-Madriga L, Salomon DR, Seron D, Sheaff M, Weening JJ: Banff '05 Meeting Report: differential diagnosis of chronic allograft injury and elimination of chronic allograft nephropathy ('CAN'). Am J Transplant 2007;7:518-526.

-2 Racusen LC, Solez K, Colvin RB, Bonsib SM, Castro MC, Cavallo T, Croker BP, Demetris AJ, Drachenberg CB, Fogo AB, Furness P, Gaber LW, Gibson IW, Glotz D, Goldberg JC, Grande J, Halloran PF, Hansen HE, Hartley B, Hayry PJ, Hill CM, Hoffman EO, Hunsicker LG, Lindblad AS, Yamaguchi Y, et al: The Banff 97 working classification of renal allograft pathology. Kidney Int 1999;55:713723.

-3 Maryniak RK, First MR, Weiss MA: Transplant glomerulopathy: evolution of morphologically distinct changes. Kidney Int 1985; 27:799-806.

4 Trpkov K, Campbell P, Pazderka F, Cockfield S, Solez K, Halloran PF: Pathologic features of acute renal allograft rejection associated with donor-specific antibody. Analysis using the Banff grading schema. Transplantation 1996;61:1586-1592.
5 Sis B, Campbell PM, Mueller T, Hunter C, Cockfield SM, Cruz J, Meng C, Wishart D, Solez K, Halloran PF: Transplant glomerulopathy, late antibody-mediated rejection and the ABCD tetrad in kidney allograft biopsies for cause. Am J Transplant 2007;7: 1743-1752.

6 El Kossi M, Harmer A, Goodwin J, Wagner B, Shortland J, Angel C, McKane W: De novo membranous nephropathy associated with donor-specific alloantibody. Clin Transplant 2008;22:124-127.

7 Gloor JM, Sethi S, Stegall MD, Park WD, Moore SB, DeGoey S, Griffin MD, Larson TS, Cosio FG: Transplant glomerulopathy: subclinical incidence and association with alloantibody. Am J Transplant 2007;7:21242132.

8 Sijpkens YW, Joosten SA, Wong MC, Dekker FW, Benediktsson H, Bajema IM, Bruijn JA, Paul LC: Immunologic risk factors and glomerular c4d deposits in chronic transplant glomerulopathy. Kidney Int 2004;65:24092418.

9 Racusen LC, Halloran PF, Solez K: Banff 2003 Meeting Report: new diagnostic insights and standards. Am J Transplant 2004; 4:1562-1566.

10 Regele H, Bohmig GA, Habicht A, Gollowitzer D, Schillinger M, Rockenschaub S, Watschinger B, Kerjaschki D, Exner M: Capillary deposition of complement split product c4d in renal allografts is associated with basement membrane injury in peritubular and glomerular capillaries: a contribution of humoral immunity to chronic allograft rejection. J Am Soc Nephrol 2002;13:23712380 .
1 Regele H, Exner M, Watschinger B, Wenter C, Wahrmann M, Osterreicher C, Saemann MD, Mersich N, Horl WH, Zlabinger GJ, Bohmig GA: Endothelial c4d deposition is associated with inferior kidney allograft outcome independently of cellular rejection. Nephrol Dial Transplant 2001;16:20582066.

12 Gloor JM, Cosio F, DeGoey S, Winters JL, Moore SB, Lager JL, Stegall MD: Long term outcome following anti-human globulin-enhanced complement dependent cytotoxicity (AHG-CDC) positive crossmatch (+XM) kidney transplantation. Am J Transplant 2008;8:182.

13 Hourmant M, Cesbron-Gautier A, Terasaki PI, Mizutani K, Moreau A, Meurette A, Dantal J, Giral M, Blancho G, Cantarovich D, Karam G, Follea G, Soulillou JP, Bignon JD: Frequency and clinical implications of development of donor-specific and non-donorspecific HLA antibodies after kidney transplantation. J Am Soc Nephrol 2005;16: 2804-2812.

14 Fotheringham J, Shrestha B, Harmer AW, McKane WS: Proteinuria precedes donorspecific alloantibody after kidney transplantation. J Am Soc Nephrol 2007;18:31A.

15 Joosten SA, Sijpkens YW, van Ham V, Trouw LA, van der Vlag J, van den Heuvel B, van Kooten C, Paul LC: Antibody response against the glomerular basement membrane protein agrin in patients with transplant glomerulopathy. Am J Transplant 2005;5:383393. 
16 Harvey SJ, Miner JH: Revisiting the glomerular charge barrier in the molecular era. Curr Opin Nephrol Hypertens 2008;17:393398.

- 17 Setoguchi K, Ishida H, Shimmura H, Shimizu T, Shirakawa $H$, Omoto K, Toki D, Iida S, Setoguchi S, Tokumoto T, Horita S, Nakayama H, Yamaguchi Y, Tanabe K: Analysis of renal transplant protocol biopsies in $\mathrm{ABO}$ incompatible kidney transplantation. Am J Transplant 2008;8:86-94.

- 18 Koch M, Broecker V, Heratizadeh A, Doege C, Strehlau J, Mengel M, Nashan B: Induction of chronic renal allograft injury by injection of a monoclonal antibody against a donor MHC Ib molecule in a nude rat model. Transpl Immunol 2008;19:187-191.
19 Smith RN, Kawai T, Boskovic S, Nadazdin O, Sachs DH, Cosimi AB, Colvin RB: Four stages and lack of stable accommodation in chronic alloantibody-mediated renal allograft rejection in Cynomolgus monkeys. Am J Transplant 2008;8:1662-1672.

20 Burns JM, Cornell LD, Perry DK, Pollinger HS, Gloor JM, Kremers WK, Gandhi MJ, Dean PG, Stegall MD: Alloantibody levels and acute humoral rejection early after positive crossmatch kidney transplantation. Am J Transplant 2008;8:2684-2694.

21 Theruvath TP, Saidman SL, Mauiyyedi S, Delmonico FL, Williams WW, Tolkoff-Rubin N, Collins AB, Colvin RB, Cosimi AB, Pascual M: Control of antidonor antibody production with tacrolimus and mycophenolate mofetil in renal allograft recipients with chronic rejection. Transplantation 2001;72:77-83.
22 Schwarz C, Regele H, Huttary N, Wahrmann M, Exner M, Nagy-Bojarsky K, Kletzmayr J, Horl WH, Bohmig GA: Rescue therapy with tacrolimus and mycophenolate mofetil does not prevent deterioration of graft function in c4d-positive chronic allograft nephropathy. Wien Klin Wochenschr 2006;118:397-404.

$>23$ Billing H, Rieger S, Ovens J, Susal C, Melk A, Waldherr R, Opelz G, Tonshoff B: Successful treatment of chronic antibody-mediated rejection with IVIg and rituximab in pediatric renal transplant recipients. Transplantation 2008;86:1214-1221.

\title{
Editorial Comment
}

\author{
M. El Nahas, Sheffield
}

The minireview by Fotheringham and colleagues from the Sheffield Kidney Institute is a comprehensive and elegant update on TG. As stated in the review, this term is now used to refer to the now obsolete one of chronic allograft nephropathy, placing more emphasis on glomerular changes and pathology whilst still associated with PTC changes including the deposition of the complement fragment C4d. Electron microscopy reveals basement membrane abnormalities in glomerular and peritubular capillaries, indicating that this is a disease of the entire renal capillary network. C4d positivity suggests a humoral, anti-donor antibody-mediated injury. TG can start insidiously a few months after transplantation and become clinically apparent after several years. Recent studies demonstrated a close association between TG and anti-HLA class II antibodies. Fotheringham and his colleagues reviewed the range of available therapies for TG. Many of these remain to be of proven value. It is expected that improved understanding of TG pathogenesis will lead to more effective therapies for this form of progressive allograft damage [reviewed in 1,2]. Amongst the novel therapies that might prove effective in the fu- ture, strategies such as anti-C5 mAb inducing an accommodation state in EC may be useful. Also, co-stimulation blockade with interference with the CD40-CD154 pathway may prove effective. Interference with CD40 induces a protective phenotype (anti-inflammatory, anti-apoptotic and anti-coagulant) in EC in conjunction with complete avoidance of adaptative humoral immunity in the host [reviewed in 3]. It is likely that this area of nephrology and immunology will see major advances over the next few years.

\section{References}

Cosio FG, Gloor JM, Sethi S, Stegall MD: Transplant glomerulopathy. Am J Transplant 2008;8:492-496.

-2 Najafian B, Kasiske BL: Chronic allograft nephropathy. Curr Opin Nephrol Hypertens 2008;17:149-155.

-3 Cruzado JM, Bestard O, Grinyó JM: Control of anti-donor antibody production post-transplantation: conventional and novel immunosuppressive therapies; in Remuzzi G, Chiaramonte S, Perico N, Ronco C (eds): Humoral Immunity in Kidney Transplantation. Contrib Nephrol. Basel, Karger, 2009, vol 162, pp 117-128. 